\title{
Taylor series expansion of nonlinear integrodifferential equations
}

\author{
${ }^{1}$ Eke A. N. and ${ }^{2}$ Jackreece P. C. \\ ${ }^{1}$ Department of Mathematics, University of Nigeria, Nnsuka \\ ${ }^{2}$ Department of Mathematics/Statistics, University of Port Harcourt, Choba Port Harcourt
}

\begin{abstract}
In this study a Taylor's method is developed to find an approximate solution for initial value problem for nonlinear integro-differential equations of the Fredholm type. The method transforms the nonlinear integro-differential equation to a matrix equation which corresponds to a system of nonlinear equations with unknown coefficients.
\end{abstract}

Keywords: Integro-differential equations, Taylor's approximation, Fredholm equation.

\section{INTRODUCTION}

Finding exact solutions of nonlinear integrodifferential equations are usually difficult to solve analytically. Since many physical problems are modelled by integral and integro-differential equations, the numerical solutions of such equations have been highly studied by many authors (see, Avudainayagam and Vani (2000), Goldfine (1977), Rashed (2003), Sezer (1994), Kythe and Puri (2002), Kanwal and Liu (1989)). Numerous works have been focusing on the development of more advanced and efficient methods for solving integral and integrodifferential equations. El-Sayed and Abdel (2003) did a comparative study of the approximate solution of integro-differential equation using Adomian decomposition method which is a semi-analytic technique and Wavelet-Galerkin's method.

In Avudainayayam and Vani (2000), Mahmoudi (2005), the numerical solution of the nonlinear integral equation was computed using WaveletGalerkin method. Here the continuous Legendre wavelets constructed on the interval $[0,1]$ is used to solve the nonlinear Volterra and integral equation of the second kind. The nonlinear part of the integral is approximated by Legendre wavelets, and the system of integral equation is reduced to a system of nonlinear equations.

In recent years there has been an increasing interest in Taylor's series solution of integral and integrodifferential equations. Yalcinbas (2002), Maleknejad and Mahmoudi (2003), Kanwal and Liu (1989), Darania and Ebadian (2006), Sezer (1994), all developed a Taylor's expansion approach to find the approximate solution for nonlinear integral and integro-differential equations. They transformed the nonlinear integral and integro-differential equation to a matrix which corresponds to a system of nonlinear equations.

However Rashed (2003), used the Lagrange interpolation method to compute the numerical solutions of differential and integro-differential equation while in Hosseini and Shahmoral (2003), Tan method is used to find the approximate solution of Fredholm integrodifferential equation with arbitrary polynomial bases. All these methods require more efforts to achieve the result and are usually developed for special types of integro-differential equations.

In this paper we consider solving a nonlinear integrodifferential equation (1) by adopting the basic ideas of the works of Maleknejad and Arzhang (2006), Darania and Ebadian (2006), Maleknejad and Mahmoudi (2003).

Consider the nonlinear integrodifferential system of the form

$$
u(x) y^{\prime}(x)=f(x)+\int_{a}^{b} K(x, t,) g(t, y(t)) d t \quad a \leq x \leq b
$$

with initial condition $y(0)=y_{0}$

Where $a, \quad b \quad$ are constants, $u(x), f(x), K(x, t), g(t, y(t))$ are known functions and $y(x)$ is the solution to be determined. We assume that the functions $u(x), f(x), K(x, t), g(t, y(t))$ are continuous and are $\mathrm{n}+1$ time continuously differentiable on the interval [a, b],

$$
g(t, y(t)), K(x, t) \in[a, b] \text { and } u(x), f(x) \in L_{2}[a, b] \text {. }
$$


Let the solution of (1) be expressed in terms of Taylor polynomial as

$y(x)=\sum_{n=0}^{N+1} \frac{1}{n !} y^{(n)}(0) x^{n}$,

which is a Taylor polynomial of degree $\mathrm{N}+1$ at the point of expansion $x=0$ and $y^{(n)}(0)$ are the coefficients to be determined.

Matrix representation of components: Let us rewrite (1) as in , Maleknejad and Arzhang (2006), Maleknejad and Mahmoudu (2003) as

$D(x)=I(x)$ where $D(x)=u(x) y^{\prime}(x)$ is called the differential part and

$$
I(x)=f(x)+\int_{a}^{b} K(x, t) g(t, y(t)) d t
$$

Is called the integral part of equation (1). We need to convert the differential part $D(x)$ and the integral part $I(x)$ to matrix form. Differentiating (2) n-times w.r.t $x$ we obtain

$D^{n}(x)=I^{n}(x)$

\subsubsection{MATRIX REPRESENTATION OF THE}

\section{DIFFERENTIAL PART}

The differential part $D^{n}(x)$ can be written as

$$
\begin{aligned}
& D^{n}\left(u(x) y^{\prime}(x)\right)=u D^{n}\left(y^{\prime}\right)+{ }^{n} C_{1} D u \cdot D^{n-1}\left(y^{\prime}\right)+{ }^{n} C_{2} D^{2} u \cdot D^{n-2}\left(y^{\prime}\right)+\ldots+{ }^{n} C_{n-1} D^{n-1} u \cdot D y^{\prime}+y^{\prime} D^{n} u \\
& (n=0,1, \ldots, N) \\
& D^{(n)}\left(u(x) y^{\prime}(x)\right)=u D^{n+1}(y)+{ }^{n} C_{1} D u \cdot D^{n} y+{ }^{n} C_{2} D^{2} u \cdot D^{n-1} y+\ldots+{ }^{n} C_{n-1} D^{n-1} u \cdot D^{2} y+D y \cdot D^{n} u \\
& \quad(n=0,1, \ldots, N)
\end{aligned}
$$

Using Leibnitz's rule, for the $\mathrm{j}^{\text {th }}$ term $(0 \leq j \leq N)$ of the components of (5) at $\mathrm{x}=0$ we obtain

$$
\begin{aligned}
& {\left[u_{l j}(x) y^{l}(x)\right]_{x=0}^{n+1}=\sum_{k=0}^{n+1}\left(\begin{array}{l}
n+1 \\
k
\end{array}\right) U_{l}^{n-k}(0) Y_{l}^{k+l}(0)} \\
& \text { for } n=0,1, \ldots, N, l=0,1, \ldots, m, N \geq m \text {, then } \\
& {\left[\left[u_{l}(x) y^{(l)}(x)\right]^{0},\left[u_{l}(x) y^{l}\right]^{(1)}, \ldots,\left[u_{l}(x) y^{l}(x)\right]^{(N)}\right]_{x=0}^{T}=U_{l} Y_{l}}
\end{aligned}
$$

where $U_{l}$ is an $(N+1) \times(N+1)$ matrix and is obtains as

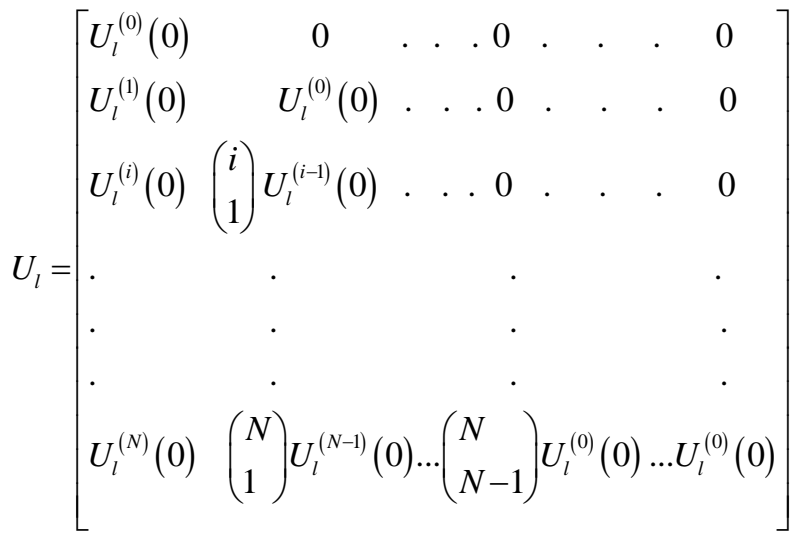


And

$Y_{l}=\left[y^{(j)}(0), y^{(j+1)}(0), \ldots, y^{(N+1)}\right]^{T}$

Let us define new matrices $\tilde{Y}_{l}$ and $\tilde{U}_{l}$ using $U_{l}$ in (7) as

$\tilde{U}_{l}=\left[A_{l}\left|U_{l}\right| B_{l}\right]$

Where $\quad A_{j}$ and $B_{j}$ are $(N+1) \times(m-j)$ matrices with zero elements respectively. Also with (8) we define

$\tilde{Y}=\left[y^{(0)}(0), y^{(1)}(0), \ldots, y^{(N)}(0), y^{(N+1)}(0), \ldots, y^{(N+m)}\right]^{T}$

Now we can construct the differential part of (2) at $x=$ 0 in matrix form

$\left[D^{(0)}(0), D^{(0)}(0), \ldots, D^{(N)}(0)\right]^{T}=\wp \tilde{Y}$

Where

$\wp=\sum_{l=0}^{m} \tilde{U}_{l}$

1.2.2. MATRIX REPRESENTATION OF THE INTEGRAL PART

According to Darania and Ebadian (2006) $I^{n}(x)$ can be written as

$I^{n}(x)=f^{n}(x)+F^{n}(x)$

where

$F^{n}(x)=\frac{\partial^{n}}{\partial x^{n}}\left(\int_{a}^{b} K(x, t) g(t, y(t)) d t\right)$

We set $g(t, y(t))=G(y(t))$ where $\mathrm{G}$ is a known smooth function with $G(y(t))$ nonlinear in $y(t)$. The Taylor expansion of $G(y(t))$ at $y(t)=0$ is given as

$G(y(t))=\left.\sum_{l=0}^{N} \frac{1}{l !} \frac{d^{l}}{d y^{l}} G(y)\right|_{y=0} y^{l}(t)$
Let

$$
W(l)=\left.\frac{i}{l !} \frac{d^{l}}{d y^{l}} G(y)\right|_{y=0} \quad \text { and } \quad y^{l}(t)=Y_{l}
$$

Then equation (11) can be written as

$G(y(t))=\sum_{l=0}^{N} W(l) Y_{l}(t)$

Substitution (13) into (12) we obtains

$I^{n}(x)=f^{n}(x)+\left.\sum_{l=0}^{N} W(l) \int_{a}^{b} \frac{\partial^{n} K(x, t)}{\partial x^{n}}\right|_{x=0} Y_{l}(t) d t$

For $l=0$, we have $Y_{0}(t)=1$ and for $l>0$ we obtain the Taylor expansion of $Y_{l}(t)$ at $t=0$ as in Darania and Ebadian (2006), in the following form

$Y_{l}(t)=\sum_{m=0}^{N} \frac{Y_{l}^{(m)}(0)}{m !} t^{m}$

Also if we substitute equation (16) into equation (15), we obtain

$I^{(n)}(x)=f^{(n)}+\left.\sum_{l=0}^{N} W(l) \int_{a}^{b} \frac{\partial^{n} K(x, t)}{\partial x^{n}}\right|_{x=0}\left[\sum_{m}^{N} \frac{Y_{l}^{(m)}(0)}{m !} t^{m}\right] d t$

Which can we written as

$I^{(n)}(x)=f^{(n)}(0)+\sum_{l=0}^{N} \sum_{m=0}^{N} K_{n m, l} Y_{l}^{(m)}(0)$

Where

$$
\begin{array}{r}
K_{n m, l}=\left.\frac{1}{m !} W(l) \int_{a}^{b} \frac{\partial^{n} K(x, t)}{\partial x^{n}}\right|_{x=0} t^{m} d t \\
(n, m, l=0,1, \ldots, N)
\end{array}
$$

The quantities $Y_{i}^{m}(0)$ for $m=0,1, \ldots, N \quad$ in equation (18) can be found from the condition $Y_{0}(t)=1$ and permutation relation 


$$
Y_{l}^{(m)}(0)=\left\{\begin{array}{lr}
\sum_{t_{1}+t_{2}+\ldots+t_{l}=m}\left(\begin{array}{l}
m \\
t_{1} t_{2} \ldots t_{l}
\end{array}\right) y^{\left(t_{1}\right)}(0) y^{\left(t_{2}\right)} \ldots y^{\left(t_{l}\right)} & l>0, m=0,1 \ldots N \\
0 & l=0, \quad m \neq 0 \\
1 & l=m=0
\end{array}\right.
$$

Where $\left(\begin{array}{l}m \\ t_{1} t_{2} \ldots . t_{l}\end{array}\right)=\frac{m !}{t_{1} ! t_{2} ! \ldots . t_{l} !},\left(t_{1} t_{2} \ldots t_{l}\right.$ are positive integers or zero)

As in Maleknejad and Mahmoudu (2003), Maleknejad and Arzhang (2006), Darania and Ebadian (2006), equation (18) can be put in matrix form as

$$
\wp \tilde{Y}-\sum_{l=0}^{N} K_{l} Y_{l}^{*}=F
$$

The matrix $K_{l}, F, Y_{l}^{*}$ are defined by

$$
\begin{aligned}
& F=\left[\begin{array}{ll}
f^{(0)}(0) \\
f^{(1)}(0) \\
\cdot & \\
\cdot & \\
\cdot & \\
f^{(N)}(0)
\end{array}\right]
\end{aligned}
$$

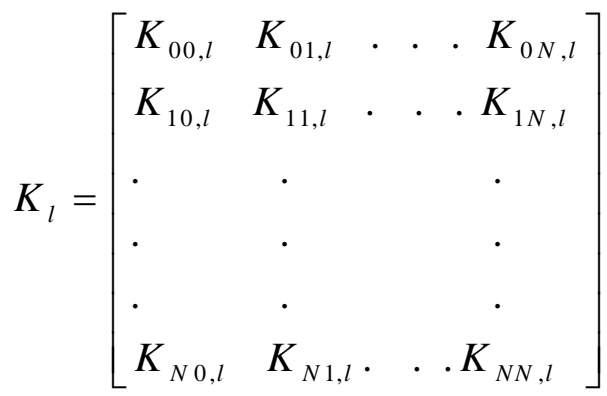

$$
Y_{l}{ }^{*}=\left[\begin{array}{l}
Y^{(0)}(0) \\
Y^{(1)}(0) \\
\cdot \\
\cdot \\
\cdot \\
Y^{(N)}(0)
\end{array}\right]
$$

From this nonlinear system, the unknown Taylor coefficients $\quad y^{(n+1)}(0)(n=0,1,2, \ldots ., N)$ are determined and substituted in (20), thus we obtain the approximate solution of the equation (1) in the form

$$
y(x) \cong \sum_{n=0}^{N+1} \frac{1}{n !} y^{(n)}(0) x^{n}
$$

\section{CONCLUSION}

Nonlinear integrodifferential equations are usually difficult to solve analytically. In many cases, it is required to obtain the approximate solution. In this study a variation of Taylor polynomial approach has been used to approximate solution of nonlinear integrodifferential equation of the fredholm type. The present method is an efficient method for the cases that the known functions have enough derivatives within the given interval. The method transforms nonlinear integrodifferential equation to a matrix equation which corresponds to a system of nonlinear equations with unknown coefficients. One of the advantages of this method is that the solution is expressed as a truncated Taylor series at $\mathrm{x}=\mathrm{c}$, then $y(x)$ can easily be evaluated for arbitrary values at low computational effort.

\section{REFERENCES}

Avudainayagam, A and Vani, C (2000), Wavelet-Galerkin method for integro-differential equations, Appl. Numer. , 32, 247-354. 
Darania,P and Ebadian, A. (2006), Development of the Taylor expansion approach for nonlinear integrodifferential equations, Int. J. Comtemp. Math. Science, Vol. 1, no. 14, 651-664.

Elsayed,S. M. and Abdel-Azizi, M. R. (2003), Acomparison decomposition method and wavelet-galerkin method for solving integro-differential equation, Appl. Math. Comput. 136, 151-159.

Goldfine, A (1977), Taylor series methods for the solution of volterra integral equations and integro-differential equations, Mathematics of computation, Vol. 31, 691707.

Hosseini, S. M. and Shahmorah, S. (2003), Tau numerical solution of Fredholm integro-differential equation with arbitrary polynomial bases, Appl. Math. Model, 27, 145-154.

Kythe, P. K.and Puri, P. (2002), Computational methods for linear integral equations, University of New Orlans, Loa 70148, USA.

Kanwal, R. P. and Liu, K. C. (1989), A Taylor expansion approach for solving integral equation, J. Math. Edu. Sci. Technol, 20, (3), 411-414.
Maleknejad, K and Mahmoudu, Y. (2003), Taylor polynomial solution of high-order nonlinear volterrafredholm integro-differential equations, Applied Mathematics and Computation, 145, 641-653.

Maleknejad, K and Arzhang, A (2006), Numerical solution of the Fredholm singular integro- differential equation with Cauchy kernel by using Taylor series expansion and Garlerkin methed, Applied Mathematics and Computation, 182, 888-897.

Mahmondi, Y. (2005), Wavelet- Galerkin method for numerical solution of nonlinear integral equation, Applied Mathematics and Computation, 167, 11191129

Rashed, M. T. (2003), Langrange interpolation to compute the numerical solutions of differential and integrodifferential equations, Appl. Math. Comput. Vol. 143, (1), 73-83.

Sezer, M. (1994), Taylor polynomial solution of volterra integral equations, Int. J. Math. Edu. Sci. Technol, 25 (5), 625-633.

Yalcinbas, S. (2002), Taylor polynomial solution of nonlinear Volterra-Fredholm integral equation, Appl. Math. Comput. 127, 195-206. 